\title{
A multistage FE updating procedure for damage identification in large-scale structures based on multiobjective evolutionary optimization
}

\author{
Ricardo Perera , Antonio Ruiz \\ Department of Structural Mechanics, Technical University, c/José Gutiérrez Abascal 2, 28006 Madrid, Spain \\ Department of Applied Mathematics, Technical University, c/Alenza 4, 28003 Madrid, Spain
}

\begin{abstract}
This study aims to develop a multistage scheme for damage detection for large structures based on experimental modal data and on finite element (FE) model updating methods applied on simple FE models. In the first stage, occurrence and approximate location of damage is performed by using damage functions in order to decrease the number of parameters to be updated. The goal in the second stage is to identify the specific damaged members and damage extent by considering only the members belonging to the regions detected as damage in the first stage. To improve identification, the optimization procedure is formulated in a multiobjective context solved by using evolutionary algorithms. Modal flexibilities and a damage location criterion dependent on frequencies and mode shapes are used as two objective functions of the multiobjective problem. The proposal is implemented in simulated case studies and in a case study of a real bridge experimentally tested with successful results.
\end{abstract}

Keywords: Multistage identification; Damage detection; Model updating; Modal flexibility; Multiobjective optimization; Evolutionary algorithms

\section{Introduction}

There has been a large volume of research devoted to vibration-based methods for damage identification in structures during recent years Changes in the physical properties of a structure result in measurable changes to the modal properties (frequencies, mode shapes and damping). But, in contrast with visual or localized experimental methods such as ultrasonic, acoustic, magnetic methods, etc. global vibration-based methods have not been generally adopted in engineering practice. However, all these localized methods require a prior knowledge of the damaged areas and, furthermore, are only applicable in accessible areas. Because of this, dynamic methods would be entirely justified in complex and large structures where a priori information of the damage location is lacking. 
Doebling

presented a review of the principal methods of damage detection based on modal parameters. Natural frequencies and mode shapes are the dynamic parameters more commonly used as damage indicators. Methods based on the measurement of natural frequencies are very attractive since this parameter can be determined by measuring at only one point of the structure However, changes in natural frequencies cannot provide spatial information about structural changes. To avoid this, mode shapes can be used, in principle, for identifying the damage location An accurate characterization of these mode shapes requires measurements at many locations, and moreover, changes in mode shapes between damaged and undamaged structures are not very significant

Derivatives of mode shapes, such as mode shape curvatures, are more sensitive to small perturbations than modal displacements, and therefore, can also be used to detect damage . However, their applicability is minimum since their estimation from experimental data are very difficult, and therefore, they are very uncertain from a statistical point of view. More recently, modal strain energy change has been directly used as a damage indicator

Other kinds of methods, based on modal flexibility, have demonstrated that this parameter is very sensitive to damage

Damage estimation techniques from modal data are often based on methods of model updating. These methods rely on a parametric model of the structure and the minimization of some objective function based on the error between the measured data and the predictions from the model The success of the finite element (FE) model updating method depends on the accuracy of the FE model, the quality of the modal tests, the definition of the optimization problem and the capability of the optimization algorithm.

However, to date, most studies performed to identify structural damage have been limited to simple analytical models or simple laboratory tests. Experiments on full-scale civil structures have been less worked with since they present many more difficulties associated with the high number of unknowns.

Most damage detection methods are based on optimization and parameter identification algorithms comprising a one-stage scheme. When these conventional methods are applied to large-scale redundant structures with many unknowns, it is not practical to identify all of the parameters in the structure at the same time since ill-conditioning and non-uniqueness in the solution of inverse problems appear as inevitable difficulties. Furthermore, a very expensive computation would be necessary.

As an alternative, damage identification based on substructuring looks attractive. In substructuring, the complete structure is divided into several substructures and the analysis is concentrated on a system with a smaller number of degrees of freedom . The basic idea contained in this procedure is based on the fact that damage occurs at critical locations on which the study should be performed. In this way, since the parameters to be identified are limited to a substructure, numerical problems can be avoided.

Multistage identification methods constitute another approach to reduce the number of variables By applying these kinds of methods, the damage identification procedure is performed in several stages. The occurrence of damage is detected in a first stage. Then, in a second stage of the identification procedure probable damaged areas are selected and, finally, the exact location and the severities of the damaged members are estimated. Using this strategy, different identification algorithms and different spatial configurations for the sensors can be adopted according to the objectives of the different stages.

In this work, a two-stage damage identification procedure formulated in a multiobjective context is proposed for large real structures. The procedure developed includes two parts or stages. The first stage is to detect the occurrence of damage and the location of damaged zones by FE model updating using damage functions The use of damage functions avoids adjusting the possible damage values of all the elements separately. This results in a reduced number of parameters to determine which contributes to avoiding optimization numerical problems and makes its application to large-scale structures easier. In the second stage, refined location of damage and estimation of its severity, an standard FE model updating technology with independent adjustment of the design variables is applied but, in this case, the number of variables is very reduced because only the elements belonging to the zones identified previously as damaged are now assumed to be damaged.

Furthermore, if it were necessary, an initial stage should be added to the two previous stages with the purpose of identifying a reference stage of the undamaged structure. This stage would be taken as a reference to perform the two-stage damage detection procedure. 
On the other hand, the choice of a suitable objective function has a big influence on the optimization problem. Usually, single-objective functions are formulated although, most times, it is really difficult to define the best objective function showing the discrepancies between the experimental and numerical modal data. In this work, as has been commented, the optimization problem in any stage of the procedure has been formulated in a multiobjective context that allows the simultaneous minimization of multiple objectives. The main purpose of performing in this way is to obtain acceptable compromise solutions for the optimization problems by trading-off the quality of fit in different groups of modal properties. In this case, two objective functions were considered. One is dependent on the modal flexibility due to its high sensitivity to damage. The other one is a function dependent on the frequencies and mode shapes and is defined in such a way that the main deficiency of the modal flexibility, provided by a certain scattering in the damage localization, is corrected. By using the multiobjective approach an improvement in damage estimations is expected to be obtained.

Furthermore, instead of traditional optimization techniques, evolutionary algorithms (EAs) have been used to obtain the set of admissible solutions for the multiobjective optimization problem, known as Paretooptimal solutions. The main motivation for using EAs to solve multiobjective optimization problems is because EAs deal simultaneously with a set of possible solutions which allows finding several members of the Pareto-optimal set in a single run of the algorithm, instead of having to perform a series of separate runs, as in the case of traditional mathematical programming techniques

The presentation of this work is organized as follows. The multiobjective model updating method used in this work is described in Section 2. It includes the way of parameterization of damage, the choice of the most suitable objective functions and the evolutionary optimization procedure used to solve the multiobjective problem. In Section 3, the multistage method is presented. The verification of the effectiveness of the proposed method is firstly illustrated in Section 4 with two numerical examples of a continuous beam and a two-story frame. The real effectiveness of the procedure is demonstrated in a real bridge dynamically tested under different levels of damage. The conclusions of this work are summarized in Section 6.

\section{Multiobjective model updating method}

FE model updating methods are based on the minimization of an objective function defined as the error between modal quantities of the measured data and model predictions. The modal properties consist of both natural frequencies and mode shapes or quantities derived from them. Updating parameters are then chosen and these are estimated by an optimization method.

Therefore, the three basic components for performing the FE model updating procedure are the formulation of the objective function, the choice of the updating parameters and the optimization technique to apply for solving the problem. A detailed discussion about each of these aspects will be conducted below.

\subsection{Parameterization of damage}

One of the key aspects of a model-based identification method is the parameterization of the updating parameters. Since optimization approaches rely on a forward model, the success of the procedure depends on the quality of the model used and the choice of the updating parameters. The model may be simple or complex. Complex approaches provide an accurate model for calculating the dynamic response of the structure with the cost of complexity and size. Furthermore, complex models require more updating parameters which might produce an ill-conditioned problem. The justification for using such a detailed model depends on the requirements of the estimation procedure and the quality of the measured data. In the case of dynamic tests in which only the lowest frequencies and their associated mode shapes are measured only a coarse model may be identified. Therefore, the use of a simple model is justified and can turn out to be more practical.

In our work, single-beam elements have been used to represent the structure. The updating parameters are the uncertain physical properties of the numerical model. It has been assumed that no alteration occurs before and after damage related to the mass, which is acceptable in most real applications. Therefore, the parameterization of the damage has been represented by a reduction factor or damage index of the element 
bending stiffness. According to Damage Mechanics , this damage index represents the relative variation of the element bending stiffness, $(E I)_{d}^{e}$, to the initial value, $(E I)^{e}$

$$
d_{e}=1-\frac{(E I)_{d}^{e}}{(E I)^{e}}
$$

This definition of a damage index $d_{e}$ for each element allows estimating not only the damage severity but also the damage location since the damage identification is then carried out at the element level. It should also be pointed out that although the damage index would take values between 0 and 1 for a damage identification procedure, their values would be included between -1 and 1 if a reference stage of the undamaged structure were to be identified, and would be considered, for this particular case, more a correction factor of the bending stiffness than a damage index strictly speaking.

\subsection{Objective functions}

Most of the model updating problems are treated with a single objective. When one is faced with the question of which criterion is suitable for damage identification, it is really difficult to define the best objective function showing the discrepancies between the experimental and numerical modal data. To perform a successful identification the parameters involved in the objective functions should be sensitive enough to the model parameters to be updated; in this case the parameters would be the damage indices, with the purpose of avoiding ill-conditioning problems.

To avoid the difficulty of having to choose the most suitable objective function, the parameter identification problem is sometimes solved by using multiple objectives . In this work, basic modal properties (modal frequencies and mode shapes) are grouped into two different objectives measuring the mismatch between those measured and the model predicted parameters.

From the literature it is concluded that the algorithms using modal flexibility, derived from frequencies and mode shapes, are very sensitive to local damage. This is the main reason for including the modal flexibility residuals as an independent objective function in this work. The flexibility matrix may be defined as

$$
[F]=[\Phi][\Lambda]^{-1}[\Phi]^{\mathrm{T}}=\sum_{i=1}^{N_{m}} \frac{1}{\omega_{i}^{2}}\left\{\Phi_{i}\right\}\left\{\Phi_{i}\right\}^{\mathrm{T}},
$$

where $[\Phi]=\left[\left\{\Phi_{1}\right\},\left\{\Phi_{2}\right\}, \ldots,\left\{\Phi_{N_{m}}\right\}\right]$ is the mode shapes matrix, $\left\{\Phi_{i}\right\}$ being the $i$ th mode shape, and [A] $=\operatorname{diag}\left(\omega_{\mathrm{i}}^{2}\right), i=1, \ldots, N_{m}$ the spectral matrix containing the eigenfrequencies of $N_{m}$ vibrating modes.

An objective function F1 depending on this parameter is formulated here using the modal assurance criterion $(M A C)[29]$ and the diagonal terms of the flexibility matrix as follows:

$$
F_{1}=1-M A C F L E X=1-\prod_{j=1}^{N_{m}} M A C F L E X_{j},
$$

where

$$
M A C F L E X_{j}=\frac{\left|\left\{F_{\text {num } j}\right\}^{\mathrm{T}}\left\{F_{\exp j}\right\}\right|^{2}}{\left(\left\{F_{\text {num } j}\right\}^{\mathrm{T}}\left\{F_{\text {num } j}\right\}\right)\left(\left\{F_{\exp j}\right\}^{\mathrm{T}}\left\{F_{\exp j}\right\}\right)} .
$$

In this equation $\left\{F_{\text {num }}\right\}$ and $\left\{F_{\text {exp } j}\right\}$ are the numerical and experimental flexibility vectors, respectively, collecting only the diagonal terms of the flexibility matrix corresponding to the $j$ th mode. The evaluation of the modal flexibility according to Eq. (2) requires the mass normalized mode shapes of the structure. Taking into account the definition of Eq. (4), the comparison between numerical and experimental flexibilities does not require any mass normalization method of measured mode shapes such as the Guyan reduced mass normalization technique.

The main advantage of using the $M A C$ criterion to formulate the objective function is the normalization of the objective function between 0 and 1 since $M A C$ takes a value of 1 when a perfect correlation between numerical and experimental values exist, while its value is 0 when no correlation exists. Furthermore, to apply the $M A C$ criterion the scaling of the experimental and numerical mode shapes does not have to be the same. 
On the other hand, the natural frequencies provide global information of the structure and they can be accurately identified through the dynamic measurements. Hence, the eigenfrequencies should be indispensable quantities to be used in the updating process. Furthermore, although very sensitive to local damage, modal flexibility provides a certain dispersion in the estimation of the damaged elements. To correct, to some extent, this deficiency as a second objective function, the modified total modal assurance criterion (MTMAC) dependent on frequencies has been adopted which is defined as follows:

$$
F_{2}=1-M T M A C=1-\prod_{j=1}^{N_{m}} M T M A C_{j},
$$

where

$$
M T M A C_{j}=\frac{M A C\left(\left\{\phi_{\mathrm{num} j}\right\},\left\{\phi_{\exp j}\right\}\right)}{1+\left|\omega_{\exp j}^{2}-\omega_{\mathrm{num} j}^{2} / \omega_{\exp j}^{2}+\omega_{\mathrm{numj} j}^{2}\right|},
$$

and where the subscripts "num" and "exp" denote the frequencies and mode shapes predicted by the numerical model and experimentally measured, respectively.

This criterion is also normalized between 0 and 1 and, therefore, it can be used perfectly combined with the $M A C F L E X$ criterion in a multiobjective optimization context.

\subsection{Strength Pareto Genetic Algorithm (SPGA) algorithm}

As has been previously described, the lack of a clear objective function in the context of real-world damage detection problems advises performing simultaneous optimizations of several objectives with the purpose of improving the robustness and performance of the procedure. Most times, from multiple objectives a singleobjective function is constructed using some weighting functions and the resultant optimization problem is solved in the usual way. The weights have a strong influence on the results and depend essentially on the accuracy of the measured data; more uncertain data should be affected by smaller weights. However, as the modeling errors and the uncertainty in the measured data are not usually known a priori, it is difficult to perform an optimal choice of the weight values. The relative importance among objectives is not generally known until the system's best capabilities are determined and trade-offs between the objectives are fully understood.

As opposed to single-objective optimization problems which accept one single-optimum solution, multiobjective optimization problems do not have a single-optimal solution, but rather a set of alternative solutions, named the Pareto-front curve, which are optimal in the sense that no other solutions in the search space are superior to them when all objectives are considered. Generating the Pareto front using weighting functions would require a family of optimization runs by varying the weights. Every combination of those weights coefficients corresponds to a single-Pareto-optimal solution.

However, by solving the parameter identification problem in the context of EAs, the need to use arbitrary weighting factors for weighting the relative importance of each objective is eliminated. Due to their characteristics, EAs, like genetic algorithms have a great potential for finding multiple optima in a single-optimization run, which is very useful in Pareto-optimization problems. These algorithms are based on a randomly generated initial population of candidate solutions in the parameter space, which by means of different genetic operators (selection, mutation and crossover) evolves towards better and better regions in the search space. The structure of the EAs has been recognized to be more appropriate to multiobjective optimization problems than that corresponding to conventional optimization techniques

Different algorithms well suited to solving the multiobjective optimization problem based on the concept of Pareto optimality have been proposed Unlike other EAs, most of these are characterized by the use of fitness sharing combined with elitism. One such algorithm, used in this work, is the SPGA. This algorithm belongs to a second generation of multiobjective EAs born out of the introduction of the notion of elitism. In the context of multiobjective optimization, elitism usually refers to the use of an external population to retain the non-dominated individuals found at each generation. For each individual in this external set, fitness is 
defined through a strength value based on Pareto dominance. This strength is proportional to the number of solutions to which a certain individual dominates. For an individual of the non-external set (i.e., the population), its fitness is calculated by adding " 1 " to the total sum of the strengths of all the external members that dominate it. With this mechanism, population diversity is maintained without any explicit sharing. Additionally, the SPGA also incorporates a clustering procedure in order to keep the size of the external set while maintaining its characteristics.

The SPGA optimization methodology proceeds in the following way:

(a) Generate an initial population of individuals $P$ representing possible candidate solutions to the problem, in our case possible values of the damage index or the correction factor in all the elements to be updated. Furthermore, create the empty external non-dominated set $P^{\prime}$.

(b) Copy non-dominated individuals from $P$ to $P^{\prime}$.

(c) Remove solutions within $P^{\prime}$ which are dominated by any other individual of $P^{\prime}$.

(d) If the number of individuals stored in $P^{\prime}$ exceeds a given maximum $N^{\prime}$, reduce $P^{\prime}$ by clustering.

(e) Evaluation of the fitness of each individual of the population in $P$ and $P^{\prime}$. Firstly, the fitness of each individual $i$ belonging to $P^{\prime}$ is calculated as

$$
f_{i}=\frac{n}{N+1} \text {, }
$$

where $n$ is the number of individuals in $P$ dominated by $i$ and $N$ is the size of $P$.

Secondly, the fitness of each individual $j$ belonging to $P$ is evaluated from the fitness of the individuals in $P^{\prime}$ as follows:

$$
f_{j}=1+\sum_{i \succ j} f_{i},
$$

where the sum is applied to all the external individuals $i \in P^{\prime}$ that dominate $j \in P$

(f) Select individuals from $P+P^{\prime}$ using a tournament selection scheme.

(g) Apply problem-specific crossover and mutation operators as usual to generate the new population.

(h) If the maximum number of generations is reached or some stop criterion is verified, then stop, or else go to Step ${ }^{\mathrm{b}}$.

\section{Multistage damage identification}

In accordance with real practice, a large structure is only instrumented in a limited way, that is, the number of sensors in which the response is measured is not very high and, therefore, the magnitudes of the mode shapes are known only at a few modal locations coincident with the location of the sensors.

This aspect, together with the high number of design variables which would result if a refined model were considered, favors the application of a multistage identification strategy. Using a hierarchical strategy, different identification algorithms and different experimental layouts can be designed in accordance with the objectives of the different stages. The strategy to adopt in the first stage consists of the detection of damage occurrence and of the rough location of the regions of the structure that contain damaged members using coarse mesh.

Once the damaged regions have been determined, the proposed strategy requires a second stage in which the specific damaged members and the damage extent should be determined by correcting only the elements at the damaged zone using a finer mesh.

\subsection{First stage: damage detection and approximate location by damage functions}

In the first stage, the damage indices associated to the locations of the set of accelerometers are defined as design variables. 
Initially, an FE mesh whose element sizes are defined by the location of the accelerometers might be considered. However, a suitable location of damage based only on the limited number of mode shape values would require damage to occur in the vicinity of one of the sensors. If damage occurs at a location between two neighboring sensors no information is available in that region to locate damage and, therefore, its detection is more difficult. To improve the ability of the identification procedure, values of mode shapes might be estimated in locations between adjacent sensors by using an interpolation scheme.

However, more locations of the mode shapes involve more elements and, therefore, according to Section 2.1, a higher number of damage variables to determine with which ill-conditioning in the solution of the inverse problem can appear Teughels et al. _ _ presented an FE updating method for damage assessment in which the number of unknown variables was reduced to obtaining a physically meaningful result, by using a set of damage functions to determine the spatial bending stiffness distribution. The updating parameters were then the multiplication factors of the damage functions. According to this procedure, the damage indices defined for each element are not considered as independent variables but rather they would be calculated by combining a limited set of global damage functions $N_{i}$ as follows:

$$
d_{e}=\sum_{i=1}^{N} N_{i}\left(x_{e}\right) p_{i}
$$

or in matrix form for the whole model:

$$
\left\{\begin{array}{c}
d_{1} \\
d_{2} \\
\vdots \\
d_{N E}
\end{array}\right\}=\left[\begin{array}{ccc}
N_{1}\left(x_{1}\right) & \ldots & N_{N}\left(x_{1}\right) \\
N_{1}\left(x_{2}\right) & \ldots & N_{N}\left(x_{2}\right) \\
\vdots & \ldots & \vdots \\
N_{1}\left(x_{N E}\right) & \ldots & N_{N}\left(x_{N E}\right)
\end{array}\right]\left\{\begin{array}{c}
p_{1} \\
p_{2} \\
\vdots \\
p_{N}
\end{array}\right\},
$$

where $N$ is the number of damage functions $(N \ll N E)$, usually coincident with the number of accelerometers, and $x_{e}$ are the coordinates of the center of element $e$. The independent variables $p_{i}$ represent multiplication factors of the damage functions and are the only unknown variables of the optimization problem. Therefore, the number of unknowns would be reduced considerably resulting in a robust optimization procedure.

The damage functions can be defined in the same way as the shape functions used in FE theory. However, those are defined on a mesh of damage elements, which in its turn is defined on top of the mesh of finite elements. In this work, linear damage functions have been used (Fig. 1). Should the nodes of the damage elements be located in the same position of the accelerometers, the variables $p_{i}$ represent damage indices at these specific locations.

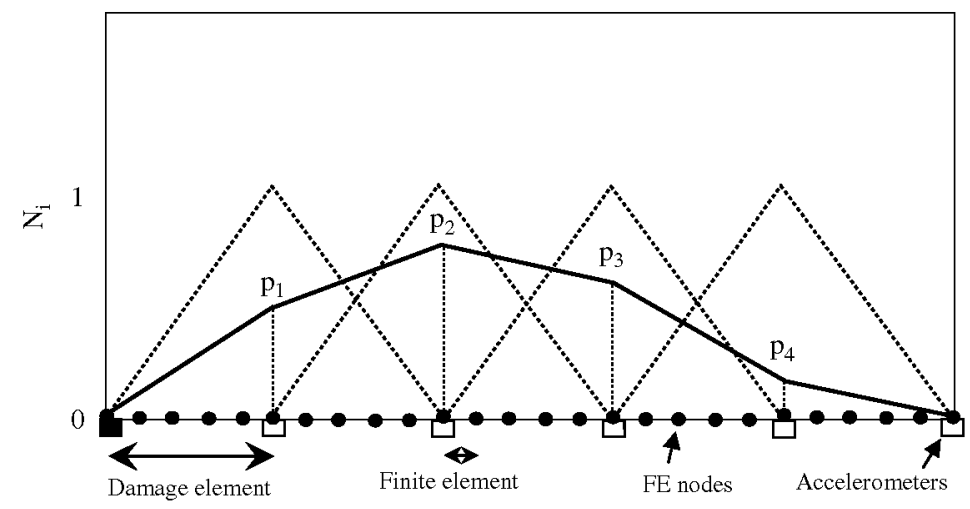

Fig. 1. Linear damage functions. 


\subsection{Second stage: identification of damaged members and estimation of severity}

In a second stage, a more detailed damage pattern and its severity can be identified. For this, it is assumed that only the elements belonging to the zones identified previously as damaged can be taken as possible damaged elements in a second updating process. The rest of the elements are considered as undamaged. In this way, the number of design variables is reduced considerably.

The procedure to be performed is as follows. Firstly, an FE coarse mesh is considered with an element size equal to the distance between accelerometers in the first stage. On this initial mesh a finer mesh is performed only in those regions identified in the previous stage as possibly damaged. From an experimental point of view, this is equivalent to installing locally more sensors within the identified regions. From an optimization point of view, the independent damage parameters to update are only considered in the elements belonging to the refined areas and, furthermore, these parameters are now defined on FEs and not on damage elements as in the previous stage. Finally, the optimization procedure is applied on the resultant FE mesh, which is not now of constant element size, taking into account that updating parameters are only defined in the refined areas.

\section{Illustrative numerical examples}

Two different types of structures were selected initially to illustrate the applicability of the proposed approach. They are a continuous beam and a two-story frame structure.

\subsection{Continuous beam}

The proposed procedure was firstly implemented to identify the location and severity in one $100 \mathrm{~m}$ long continuous beam with a box cross-section. The properties of the beam are: cross-sectional area $A=6.82 \mathrm{~m}^{2}$; moment of inertia $I=0.66273 \mathrm{~m}^{4}$; Young's modulus $E=35 \mathrm{GPa}$; density $\rho=2500 \mathrm{~kg} / \mathrm{m}^{3}$.

For the application of the first stage, identification and approximated location of damage, it was assumed that the magnitudes of the modal shapes are known only every $5 \mathrm{~m}$. From an experimental point of view, this would be equivalent to putting accelerometers in 21 points of the beam. According to this layout, 21 design variables were considered, one for each section in which modal shape was known. Therefore, the beam was divided into 20 damage elements (Fig. 2). Piecewise linear functions were used for identifying the damage distribution due to the loss of bending stiffness. In turn, each damage element was divided into twodimensional five beam elements resulting in a mesh of 100 elements.

To evaluate the damage detection ability of the proposed algorithm, one damage case was analyzed considering only one damaged element, element 32 of the beam (Fig. 2), with a severity of 0.7 . The "measured" modal responses of the beam before and after damage were generated numerically. These numerically generated measurements, instead of experimental measurements, were used to evaluate the proposed inverse procedure. Only the four lowest vibration modes were considered and, as was commented in the previous paragraph, the vertical modal displacements were read at 21 points. To check the robustness of

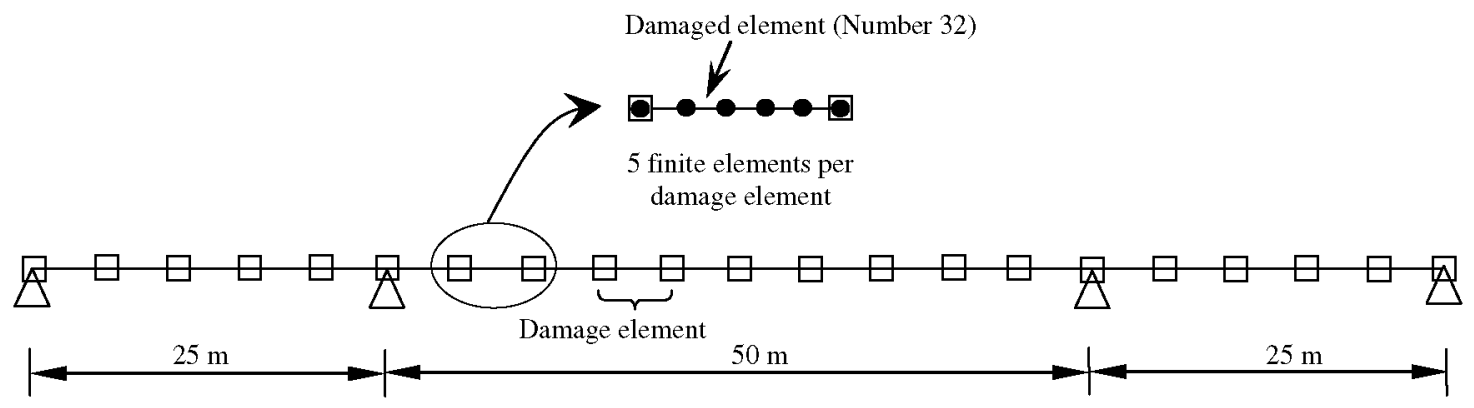

Fig. 2. First stage mesh for the continuous beam. 
the procedure, $5 \%$ artificial random noise was added to the theoretically calculated frequencies and mode shapes.

The SPGA algorithm with $M A C F L E X$ and $M T M A C$ criteria was applied with the following parameters: Size of population $=100$; single-point crossover scheme with probability of 0.8 ; probability of mutation $=0.01$. The stop criterion was set either when a maximum number of generations equal to 150 was reached or when the non-dominated set was constituted by ten or more individuals. Furthermore, on all test problems, a tolerance of 0.05 for the objective functions $(F 1 \leqslant 0.05, F 2 \leqslant 0.05)$ was chosen to limit the region of Pareto-optimal solutions.

In Fig. 3, the Pareto front is shown after three independent runs were performed. It can be observed that Pareto points clearly favor the second objective function, $M T M A C$, at the expense of the first one, $M A C F L E X$, since the values of that objective function are lower. As most of these points have a very similar value for the second function, the point with the lowest value for the first objective function was selected for the final application. Fig. 4 shows the damage predictions for this first stage by using multiobjective optimization and single-objective optimization considering $M A C F L E X$ as the only criterion. It is evident that $M T M A C$ and $M A C F L E X$ considered jointly give a clearer indication of the damage location than $M A C F L E X$ only, for which a sparsity appears. According to this, for the application of the second stage of determination of specific damaged elements and the damage severity, the only elements considered as possible damaged elements were those derived from the multiobjective procedure for which damage exceeds 0.1 , i.e. elements $26-35$.

To perform the second stage of detection, a coarse finite element mesh coincident with the damage elements of the first stage was now considered except for the 10 elements between 26 and 35 for which the original size was kept (Fig. 5). Now, it was assumed that damage could only appear in this zone, i.e. the multiobjective optimization procedure is solved with only 10 damage variables, one for each element. It is important to remark that, unlike the first stage, the design variables are now defined to the level of element, not of section. This is because in order to solve the inverse procedure in the second stage no damage functions are considered since the number of design variables has decreased after the first stage. Experimentally, the development of the second stage would be equivalent to performing local vibration measurements and using a few modal components within the identified damage region during the first stage.

The same parameters as in the first stage were used for the SPGA algorithm but a tolerance of 0.03 was now chosen to limit the region of Pareto-optimal solutions with the purpose of reaching a good estimation in the

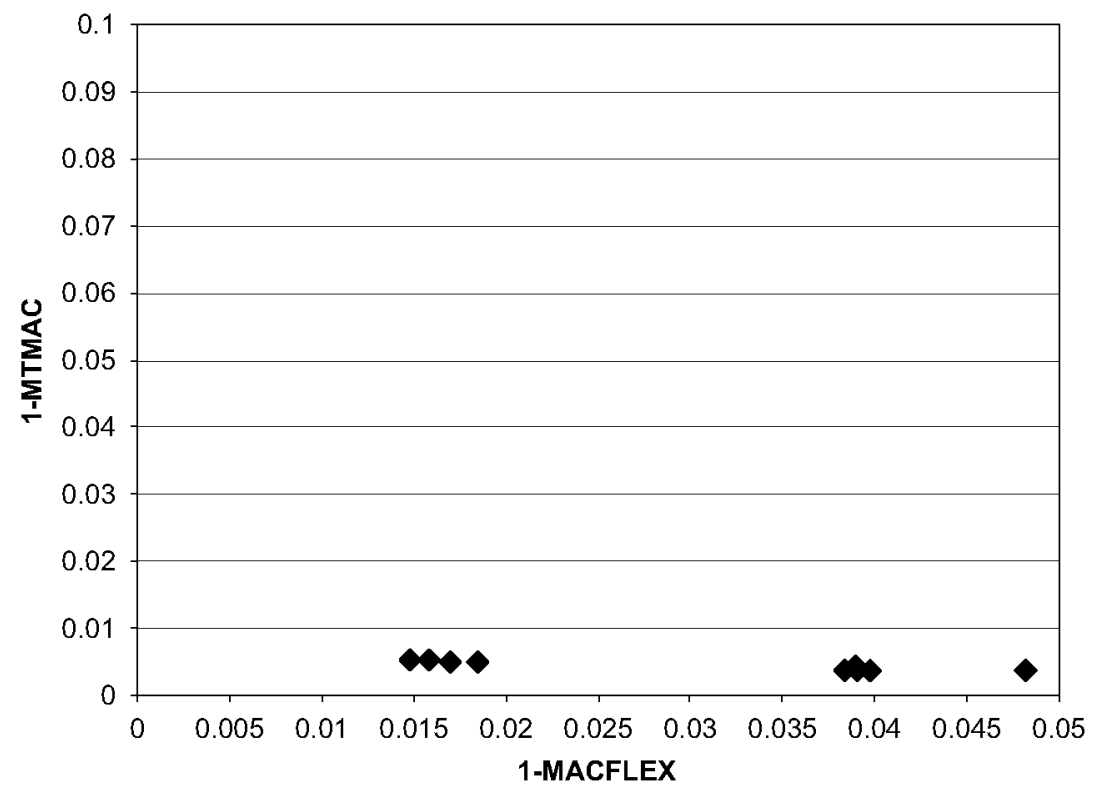

Fig. 3. Pareto-optimal solutions-continuous beam first stage. 


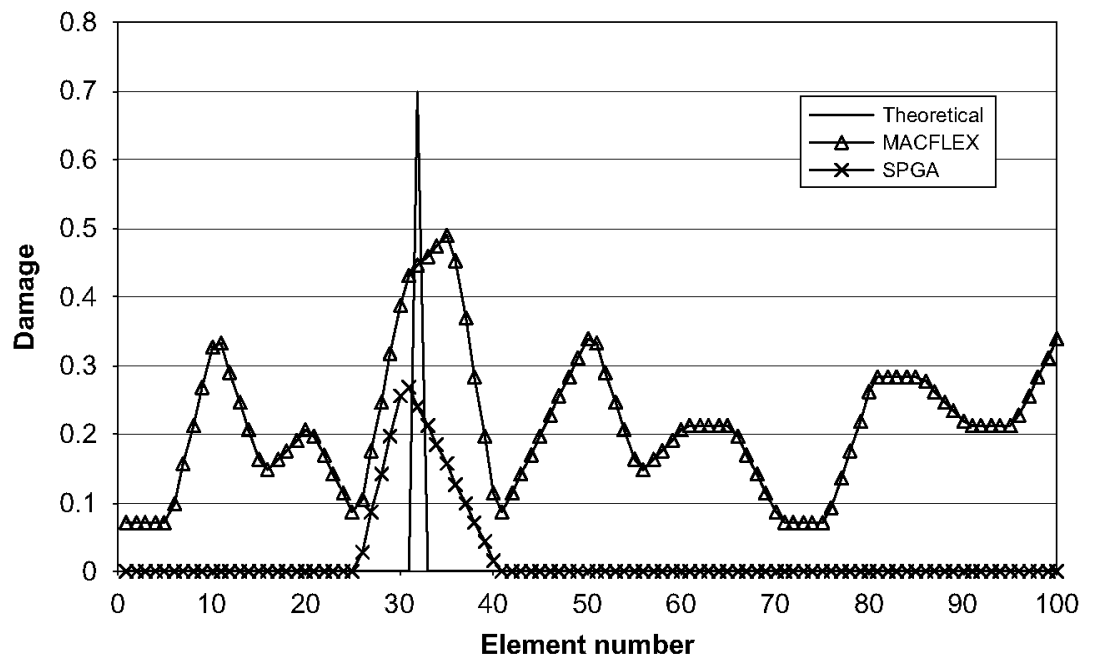

Fig. 4. Damage distribution - continuous beam first stage.

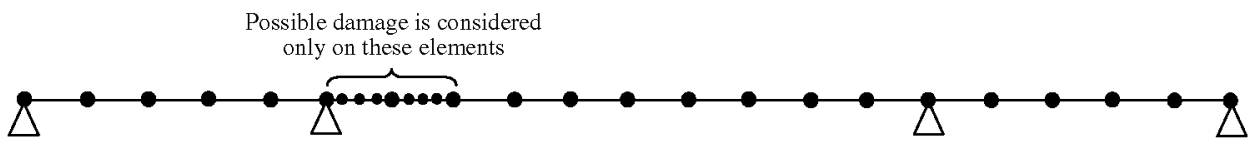

Fig. 5. Second stage mesh for the continuous beam.

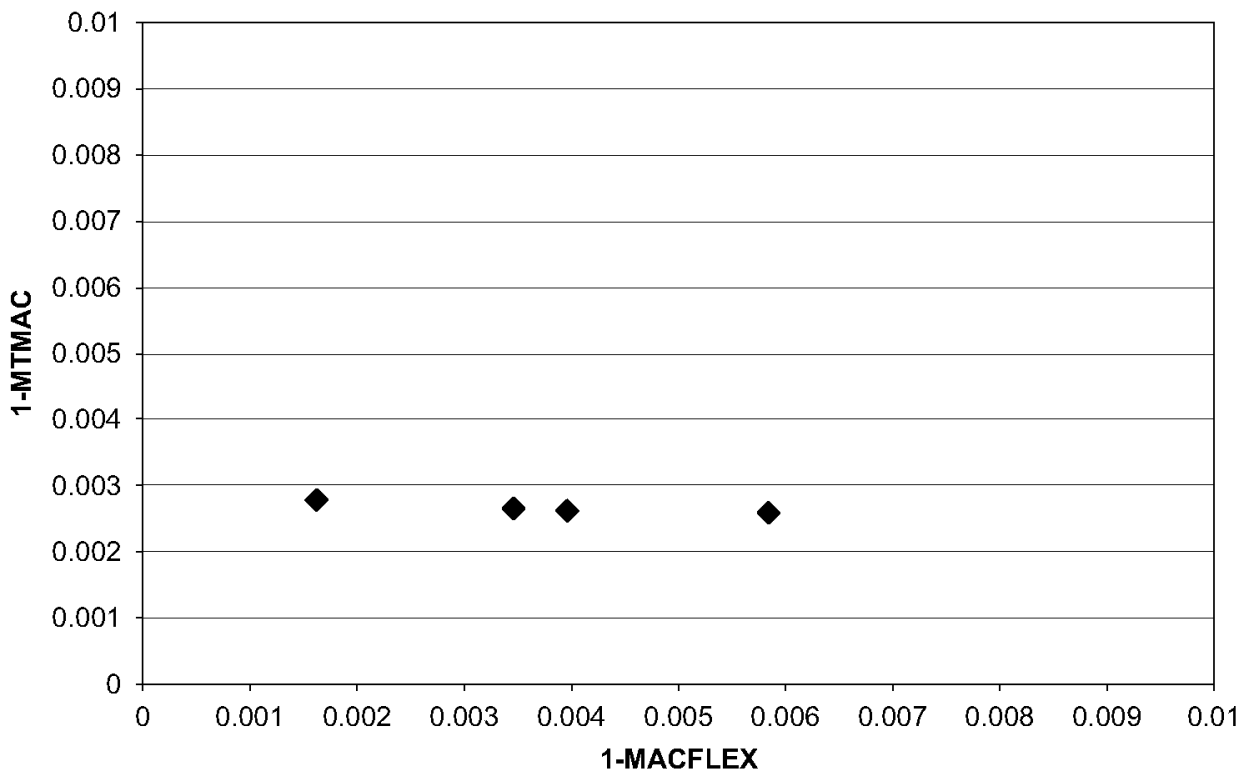

Fig. 6. Pareto-optimal solutions-continuous beam second stage.

location and severity of damage. The calculated Pareto front is shown in Fig. 6. The four points obtained correspond to very low values of the objective functions, which reveals a very good estimation. In Fig. 7, damage distribution is shown for the Pareto point with the lowest value of MACFLEX objective function. Compared to the theoretical value, the estimated location and severity is almost perfect. 


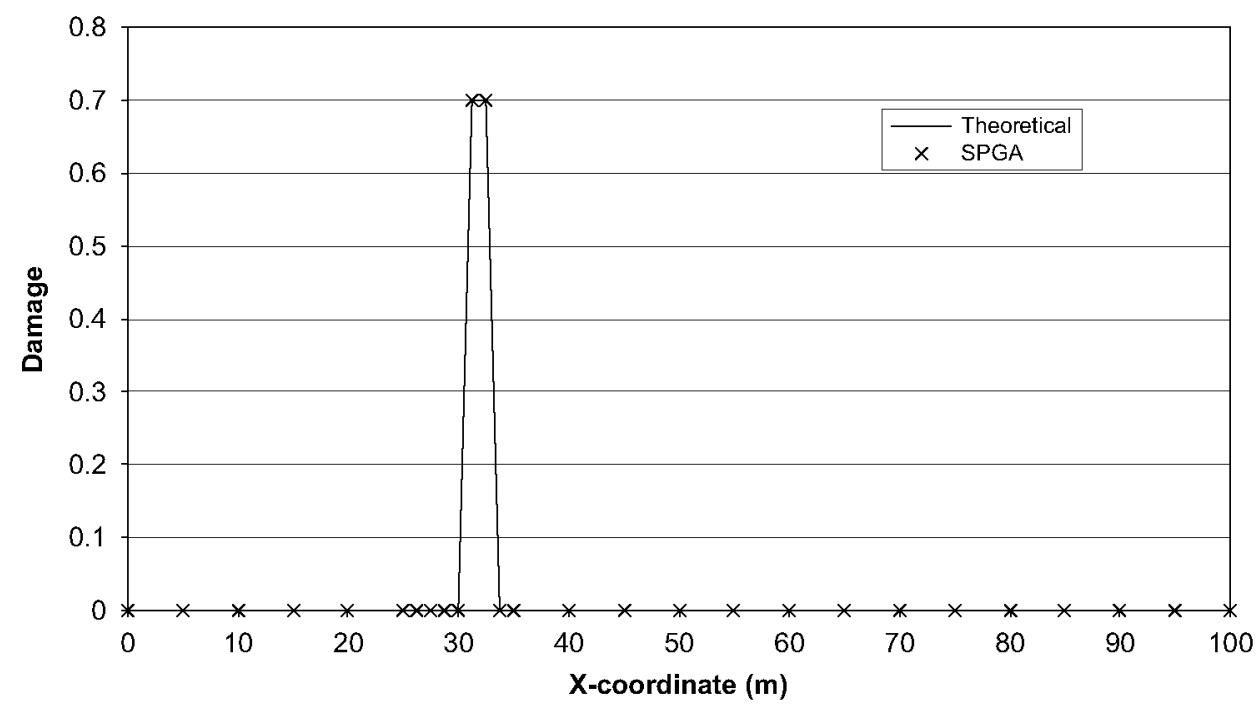

Fig. 7. Damage distribution - continuous beam second stage.

\subsection{Two-story frame}

Secondly, the multistage optimization procedure was applied to locate and quantify damage in a two-story frame with the following properties: cross-sectional area $A=0.04 \mathrm{~m}^{2}$; moment of inertia $I=1.33 \times 10^{-4} \mathrm{~m}^{4}$; Young's modulus $E=35 \mathrm{GPa}$; density $\rho=2500 \mathrm{~kg} / \mathrm{m}^{3}$.

For the application of the first stage of damage detection, it was assumed that modal displacements were known at only 18 cross-sections of the structure. This meant that only 18 independent damage variables, associated to each one of these sections, were considered for defining, therefore, 18 damaged elements (Fig. 8). Furthermore, only horizontal modal displacements were assumed to be 'measured' in the columns while for the beams only the vertical components of mode shapes were 'measured'. As rotational components of mode shapes were not considered in the calculations, in order to be consistent with experimental reality, modal displacements at intermediate cross-sections of beams and columns were included to compensate this deficiency.

The frame was modeled with 72 equal elements of $0.2 \mathrm{~m}$ in length and 72 nodes resulting in 4 FEs per damage element (Fig. 8).

To check the validity of the proposed model, a single-damage scenario was considered consisting of only one damaged element, element 12 , located adjacent to the beam column joint at the top of the first story left column (Fig. 8). A damage severity equal to 0.7 was assumed. A set of modal parameters with $5 \%$ of noise was then calculated and treated as data for the 'real' damaged structure. Only the first four modes were used to simulate incomplete modal data in the real situation.

To make an approximate prediction of the damaged regions during a first stage the same algorithm with the same parameter values and stop criterion as in the previous example was used. The Pareto-optimal set of solutions computed with the SPGA is shown in Fig. 9. As in the previous example, the Pareto points give priority to the second function and, furthermore, have a very similar value for this $f$ unction. Because of this, the point with the lowest value for the first objective function was selected to obtain the damage distribution (Fig. 10). Setting a value of 0.15 as damage threshold, damage is considered to occur only at elements $11,12,13,14,25$ and 26 , i.e., the contiguous elements to the joint in which the damaged element is located. This area constitutes the damage influence zone.

To perform the second stage of detection a coarse FE mesh coincident with the damage elements of the first stage was now considered except for the six elements detected previously as belonging to the damage influence zone for which the original size was kept (Fig. 11). Now only these six elements 


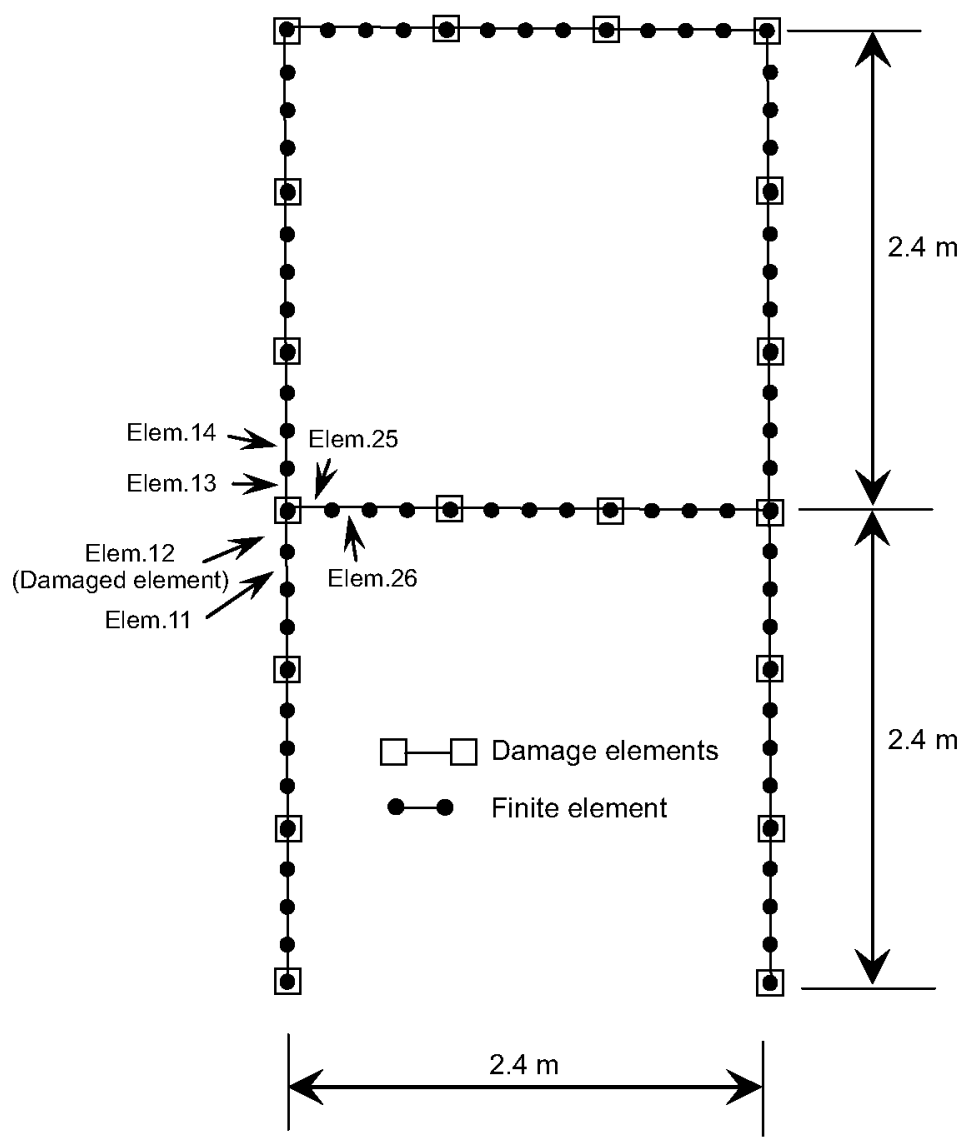

Fig. 8. First stage mesh for the two-story frame.

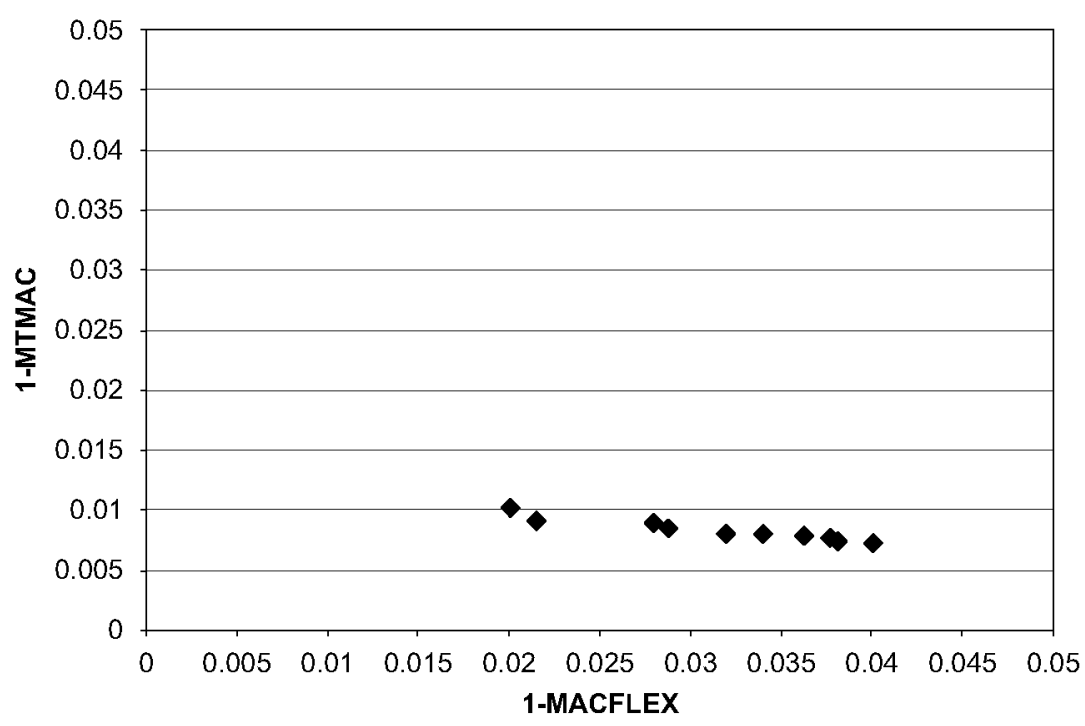

Fig. 9. Pareto-optimal solutions-two-story frame first stage.

were assumed to be possibly damaged, i.e., six independent damage variables were considered in the second stage. By applying the SPGA an almost perfect estimation for damage location and severity was obtained (Fig. 12). 


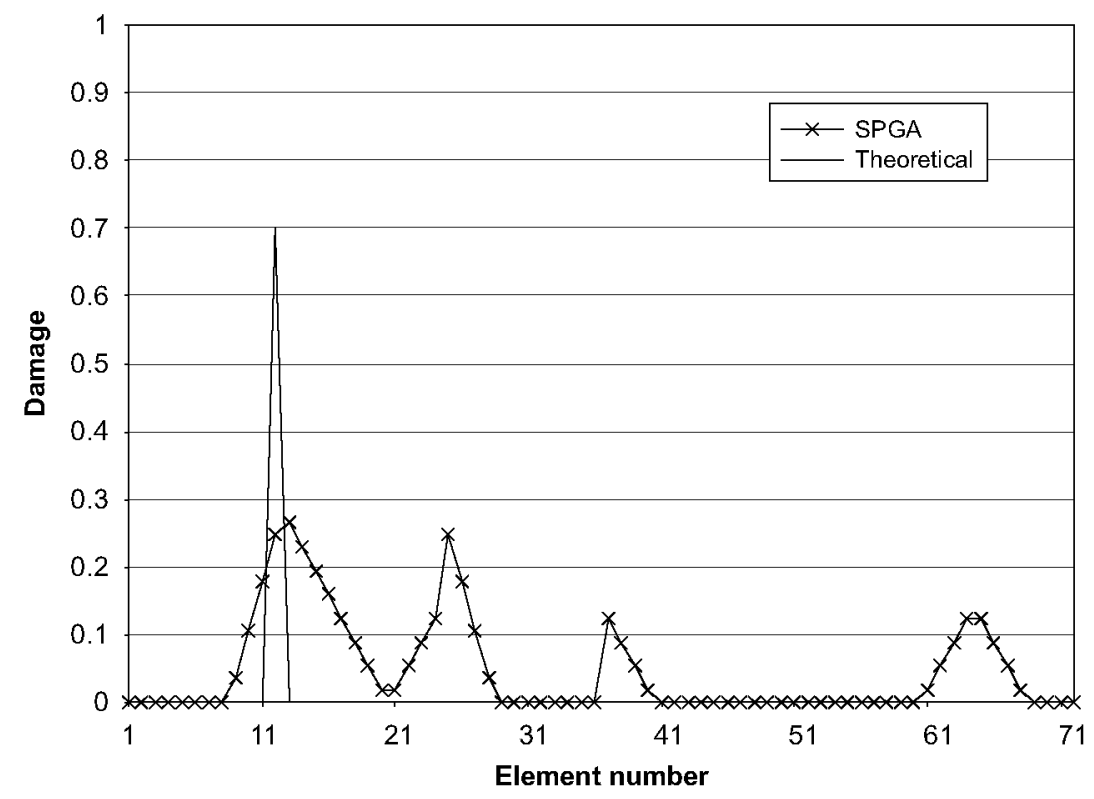

Fig. 10. Damage distribution-two-story frame first stage.

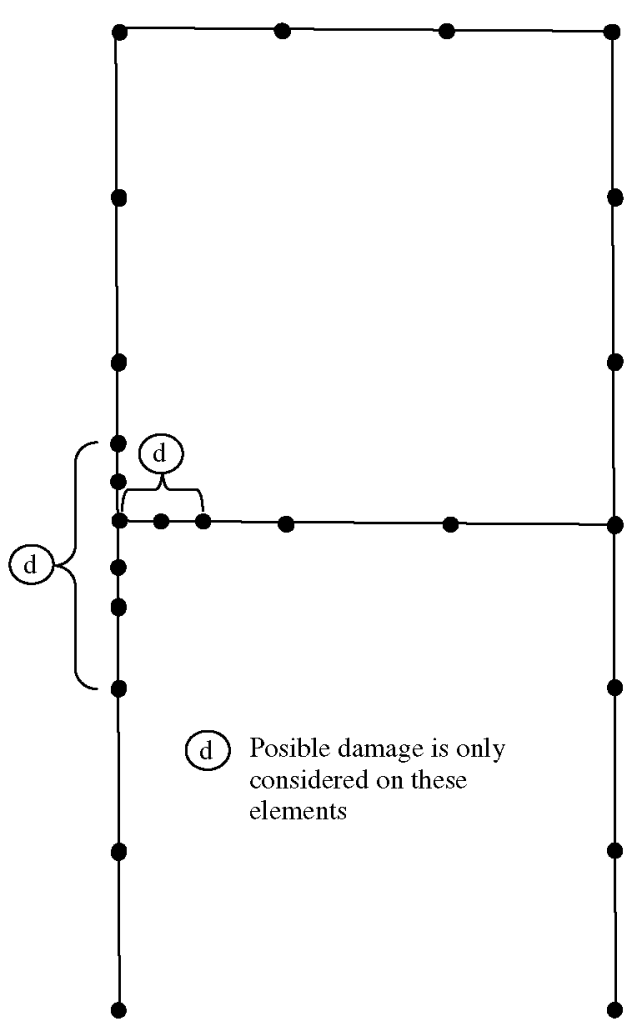

Fig. 11. Second stage mesh for the two-story frame.

To evaluate the sensitivity of the procedure to the number of measured components of the mode vectors, only one translational DOF at the midpoint of each beam/column was added to those measured at the joints (Fig. 13) during the first stage. This is equivalent to assuming 12 damage variables, one for each measured 


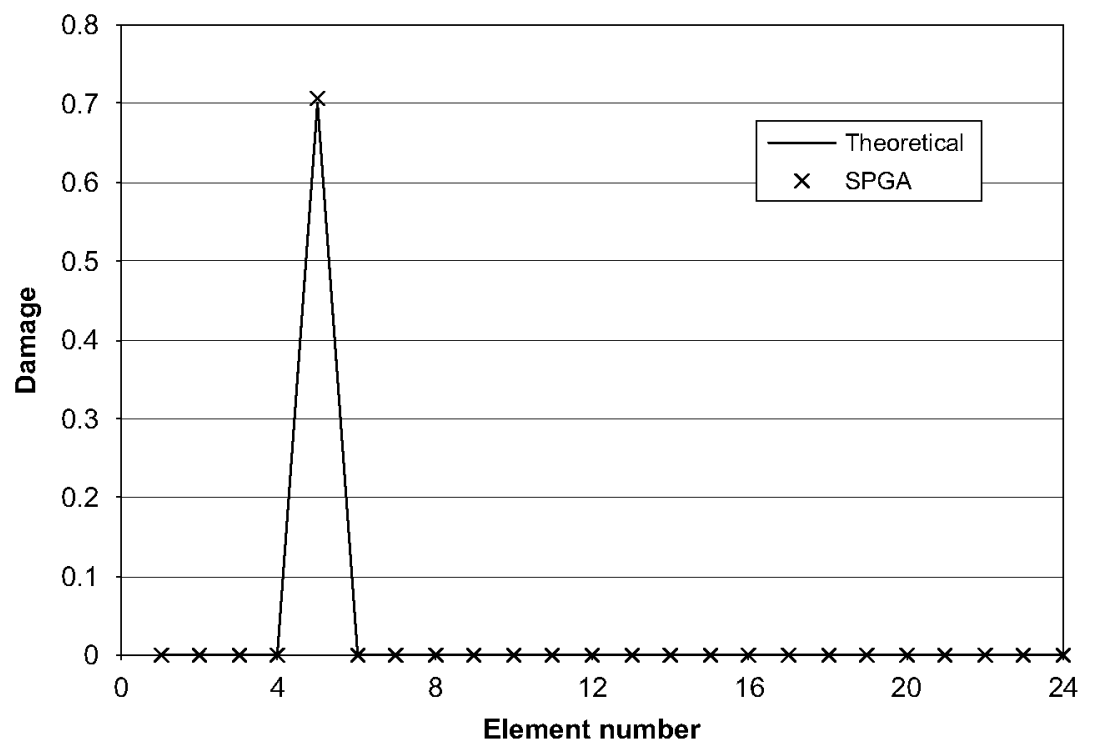

Fig. 12. Damage distribution-two-story frame second stage.

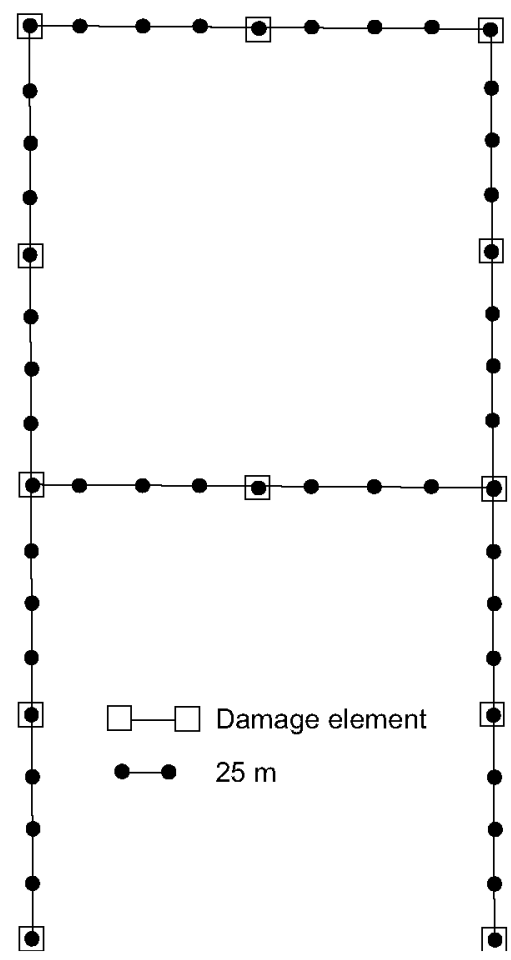

Fig. 13. Mesh for the two-story frame considering less measurement sensors.

DOF. Solving the problem for the same damage case as before, the estimation accuracy was also found to be very good. This demonstrates the effectiveness of the method even in the case of not many sensors being available. Logically, if only displacement sensors at the joints had been considered the estimations would have been very deficient. 


\section{Damage detection of $\mathbf{I}-40$ bridge}

The I-40 bridge over the Rio Grande in New Mexico (USA) was dynamically tested by Farrar et al. to investigate if modal parameters could be used to identify structural damage within the bridge. Its data set has been analyzed in the past by many different authors using different methods. With the same purpose, their experimental modal data were used in this work to evaluate the feasibility and reliability of the proposed multistage method when applied over a real structure.

The I-40 bridge consists of twin spans made up of a concrete deck supported by two welded-steel plate girders and three steel stringers. Loads from the stringers are transferred to the plate girders by floor beams. Four different levels of damage (D-1-D-4) were introduced into the bridge by gradually cutting to the middle span of the north plate girder. Damage was introduced by making various torch cuts in the web and flange of the girder as shown in Fig. 14. Unlike what was expected, frequency value increased for the first two levels of damage because the ambient temperature of the bridge played a more important role in the variation of the dynamic characteristics of the bridge than the artificially introduced damage. However, the statement changed for the third and fourth damage levels, especially for the last one, and, therefore, this last damage scenario was used in this study.

Forced vibration tests were performed when the bridge was in an undamaged state and after each of the four incremental levels of damage had been introduced. One portion of the bridge with three continuous spans $(39.9 m+49.7 m+39.9 m)$ was tested and, therefore, was considered in this study. For this, a coarse set of

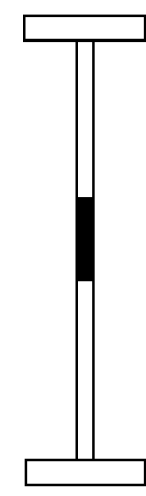

D-1

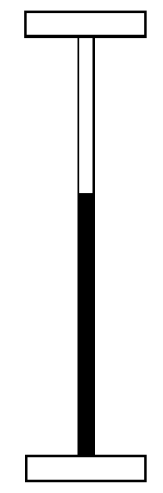

D-2

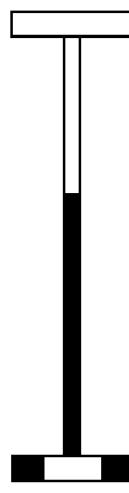

D-3

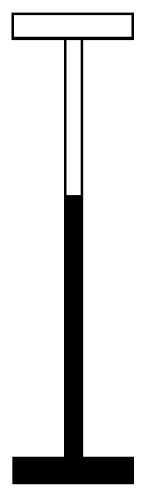

D-4

Fig. 14. Four levels of damage introduced at the girder.

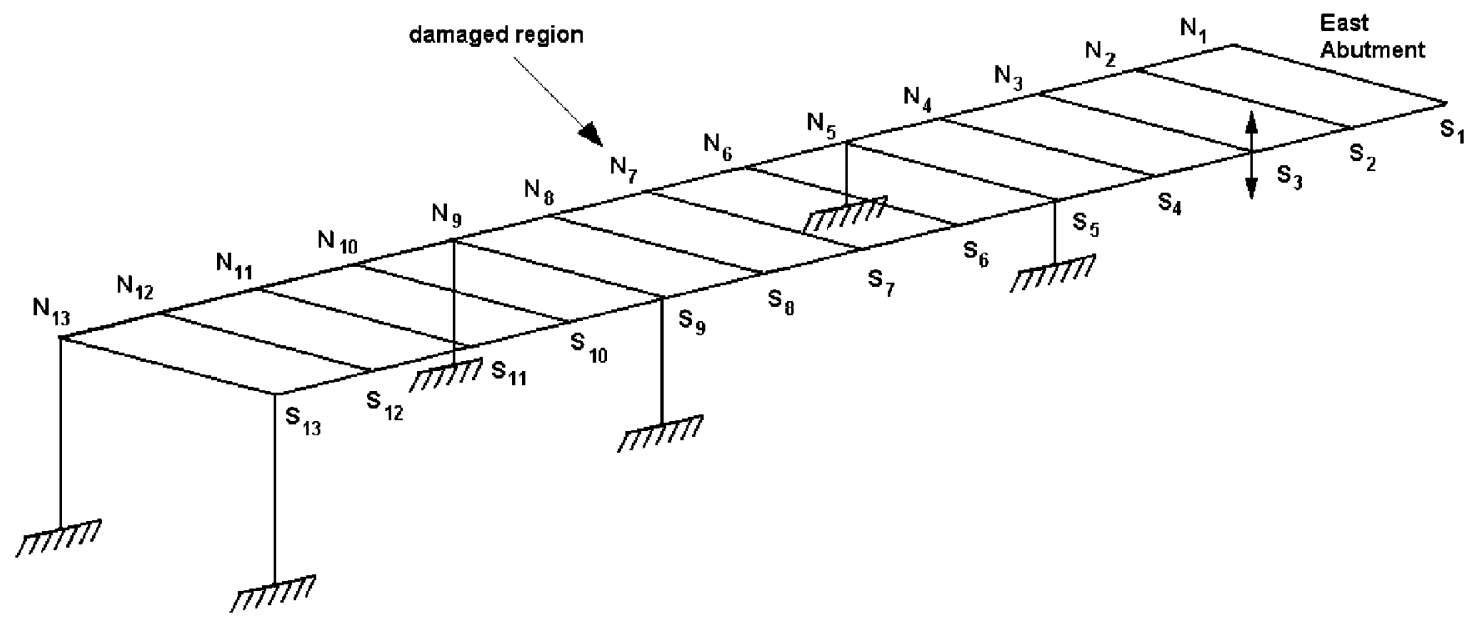

Fig. 15. Accelerometer locations. 
Table 1

Comparison of experimental and updated model modal properties of undamaged bridge I-40

\begin{tabular}{llllc}
\hline & \multicolumn{2}{l}{ Natural frequencies $(\mathrm{Hz})$} & & Error $(\%)$ \\
\cline { 2 - 5 } Mode & Experimental & Updated FE model & -1.61 & MAC $(\%)$ \\
\hline First flexural & 2.48 & 2.44 & - & -0.28 \\
First torsional & 2.96 & - & -2.7 & 99.5 \\
Second flexural & 3.5 & 4.51 & - & -12 \\
Third flexural & 4.08 & - & - & - \\
Second torsional & 4.17 & - & & - \\
Third torsional & 4.63 & & & -107 \\
\hline
\end{tabular}

measurements was firstly made with 26 accelerometers, 13 over each plate girder, mounted in the vertical direction to the inside web of the plate girder at mid-height and equally spaced in the axial direction (Fig. 15). From the first six modes of the undamaged bridge identified from the experimental data, modes 1,3 and 4 are flexural modes while modes 2, 5 and 6 are torsional modes (Table 1). A more detailed summary of the test procedures

To implement the model updating technique described previously, an appropriate parametric FE model of the bridge was considered. The developed FE model to identify damage was aimed at simulating the dynamic behavior of the bridge in a vertical direction. To do this, the deck and piles of the bridge were modeled by twodimensional beam elements and for the FE updating procedure only experimental modal displacements measured at the north plate girder (damaged girder) were considered. Equivalent values for the cross-sectional area and the bending moment of inertia of the section of the main girder and piers were pre-calculated and given as the initial inputs for the beam elements. Two sets of beam properties were developed because the plate girders have increased flange dimensions at locations near the interior columns. Fixed boundary conditions were specified at the base of the piers and the translations were restrained at the abutment. Furthermore, the nodes corresponding to the bottom of the plate girder were constrained to have the same translation in both directions as the nodes of the top of the piers to which they were connected.

The concrete was considered to be homogeneous with an initial value for the Young's modulus of $E=200 \mathrm{GPa}$.

Forty-eight beam elements were used to model the girder and 12 to model the piers and the columns.

Before applying the damage identification algorithm, a benchmarked FE model of the undamaged bridge was obtained by FE updating. To carry out the FE updating only pure bending modes were considered since only modal displacements measured at the north plated girder were used. Therefore, only 1, 3 and 4 identified modes of vibration were used for current FE model updating procedure and, furthermore, the bending stiffness of the beam elements of the girder and the piers was selected for updating. This scheme of parameterization would involve 60 correction parameters to determine, 48 accounts for the stiffness of the deck and 12 accounts for the stiffness of the piers. As the identification of a structure with many unknowns is not practical, the number of design variables was reduced using damaged elements. For this, 12 damage elements were defined for the deck and 3 for the columns; each damage element contained 4 FEs (Fig. 16). The location of each node of the damaged elements of the deck was made coincident with the location of the measurement sensors with the purpose of evaluating the objective functions to minimize at these points. Then, correction factors (with values between -1 and 1) were only defined at the damaged element nodes decreasing considerably their number.

Once the significant parameters were selected, the MACFLEX and MTMAC objective functions were simultaneously minimized by the SPGA algorithm using the following parameters: size of population $=100$; single-point crossover scheme with probability of 0.8 ; probability of mutation $=0.01$. The stop criterion was set either when a maximum number of generations equal to 200 was reached or when the non-dominated set was constituted by ten or more individuals. These SPGA parameter values were kept in all the optimization procedures performed on this structure. The final correlations of frequencies and mode shapes after FE model updating are shown in Table 1. The difference between FE and experimental frequencies shows an excellent 


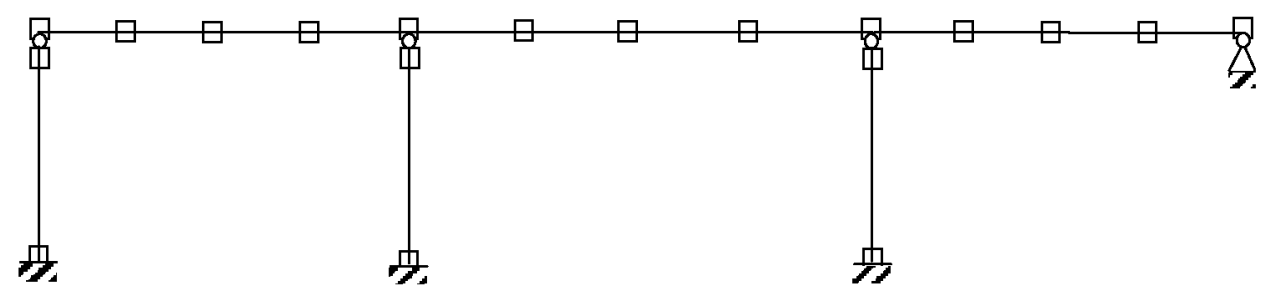

Fig. 16. Initial FE updating: damage elements mesh (each damage element contains four finite elements).
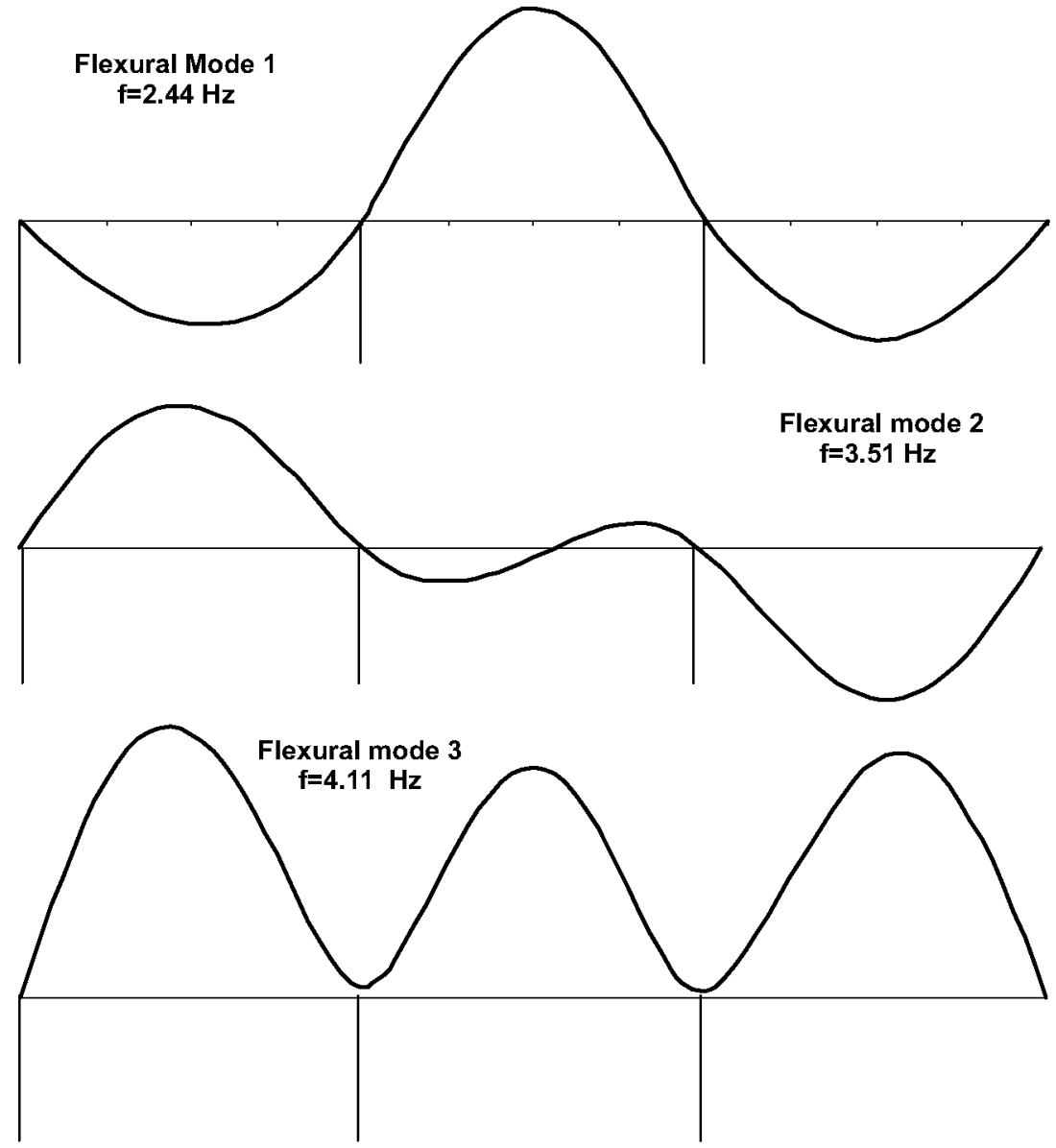

Fig. 17. First three updated numerical flexural modes.

tuning. As a guideline, all mode pairs with a $M A C$ value higher than $95 \%$ might be treated as correlated, so that the first three flexural modes (Fig. 17) would be correlated.

Once the undamaged benchmarked model was obtained, the SPGA was applied over the same mesh of FEs and damage elements as a means of approximately identifying damaged regions. To do this, the coarse set of experimental measurements performed over the bridge affected with the fourth damage level D4 was used (Fig. 14). Damage was introduced close to the intermediate section of the midspan (Fig. 15) and the fourth level can be qualified as severe. Now the design parameters to update are the damage variables, whose values are between 0 and 1 , defined at the damaged elements nodes. The magnitudes of the damage at intermediate nodal locations are estimated by interpolation using damage functions, therefore making it possible to locate 


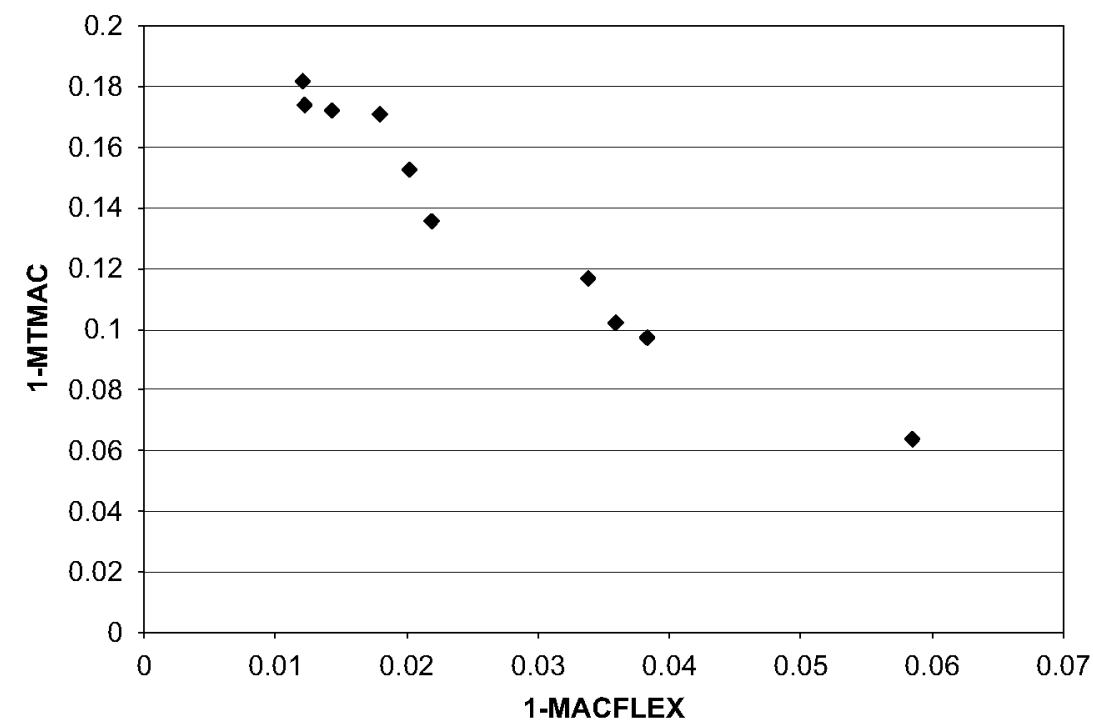

Fig. 18. I-40 bridge Pareto-optimal solutions - first stage of damage identification.

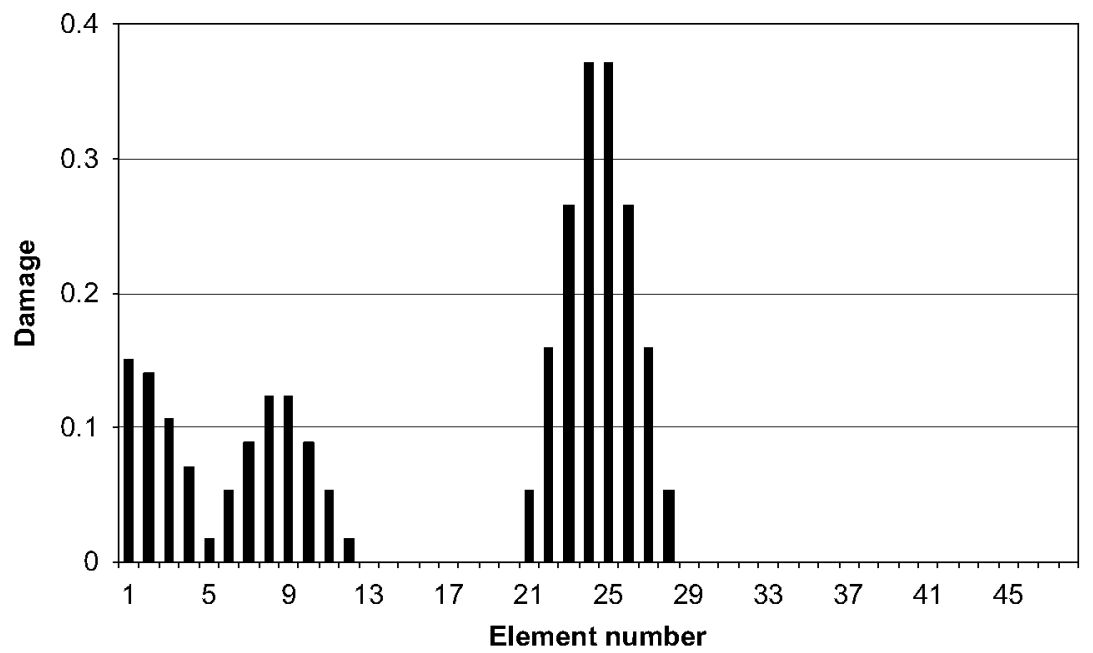

Fig. 19. Damage distribution- $-\mathrm{I}-40$ bridge first stage.

damage between adjacent sensors even if no information is available in that region. The Pareto-optimal solutions achieved by the SPGA are shown in Fig. 18. Among all the Pareto points, the one selected to represent the damage distribution is that giving the minimum value for the following expression:

$$
\sqrt{(1-M A C F L E X)^{2}+(1-M T M A C)^{2}}
$$

From the damage distribution for the deck shown in Fig. 19 corresponding to the selected point, it appears evident that damage is located at the midspan of the analyzed portion of bridge.

With the purpose of more precisely estimating the exact location and severity of damage, in a second stage, a more refined set of measurements was made near the damage location after each level of damage had been 
Table 2

Comparison of experimental and updated model modal properties of damaged bridge I-40

\begin{tabular}{llllc}
\hline & \multicolumn{2}{l}{ Natural frequencies $(\mathrm{Hz})$} & & \\
\cline { 2 - 5 } Mode & Experimental & Updated FE model & Error $(\%)$ & MAC $(\%)$ \\
\hline First flexural & 2.312 & 2.305 & -0.3 & 99 \\
First torsional & 2.844 & - & - & - \\
Second flexural & 3.5 & 3.37 & -3.71 & 97.8 \\
\hline
\end{tabular}

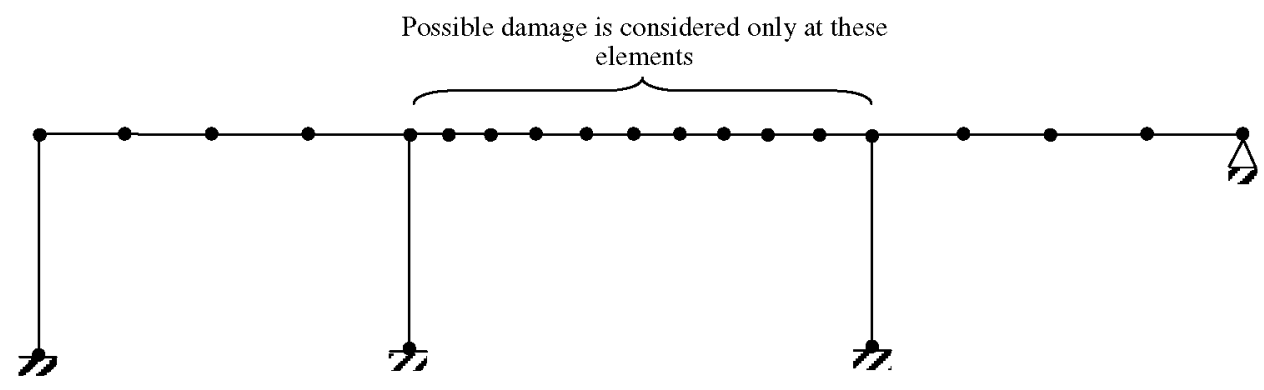

Fig. 20. FE mesh for I-40 bridge-second stage.

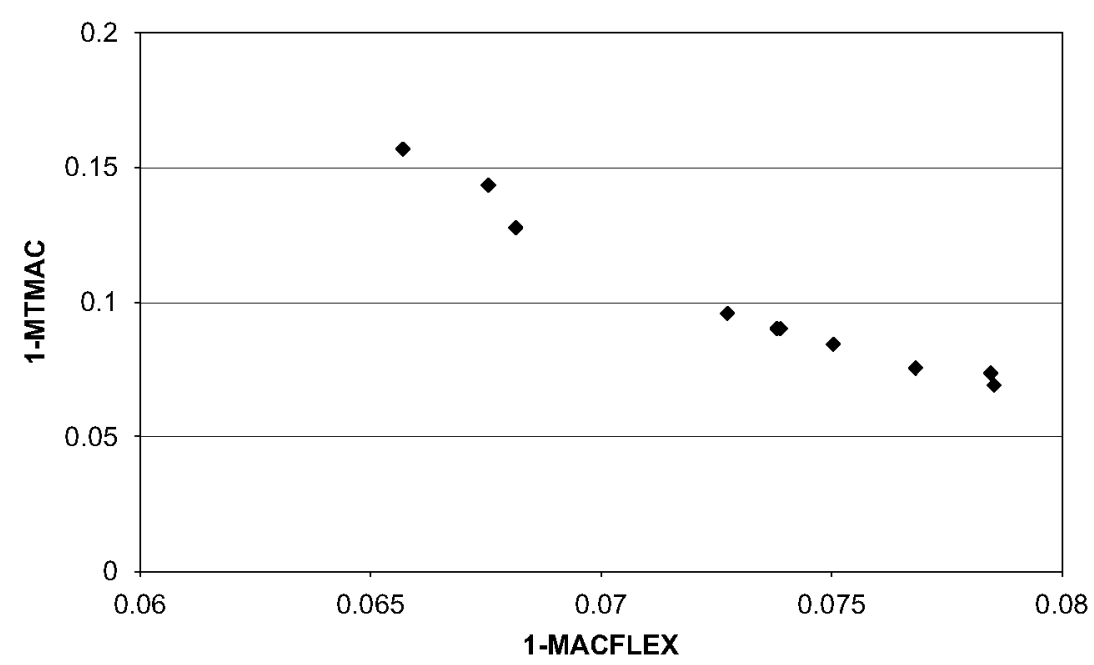

Fig. 21. I-40 bridge Pareto-optimal solutions - second stage of damage identification.

introduced using accelerometers placed in the vertical direction at a nominal spacing of approximately $0.49 \mathrm{~m}$ along the midspan of the north plated girder. The first three flexural modal frequencies are given in Table 2.

The proposed multiobjective parameter identification methodology was applied to identify damage in this second stage. For this, a mesh of 21 FEs was used (Fig. 20) but now damage was assumed to be possible only at any of the elements located at the midspan. Therefore, only 10 independent damage variables, corresponding to the $10 \mathrm{FEs}$ of the midspan, should be determined by optimization. Furthermore, nodal points at the midspan were located at the same position of the refined set of accelerometers corresponding to the experimental setup. The Pareto front and the predicted damage distribution are plotted in Figs. 21 and 22 , respectively. It is very clear that the matching between the real damage and the predicted damage is excellent for both location and severity. 


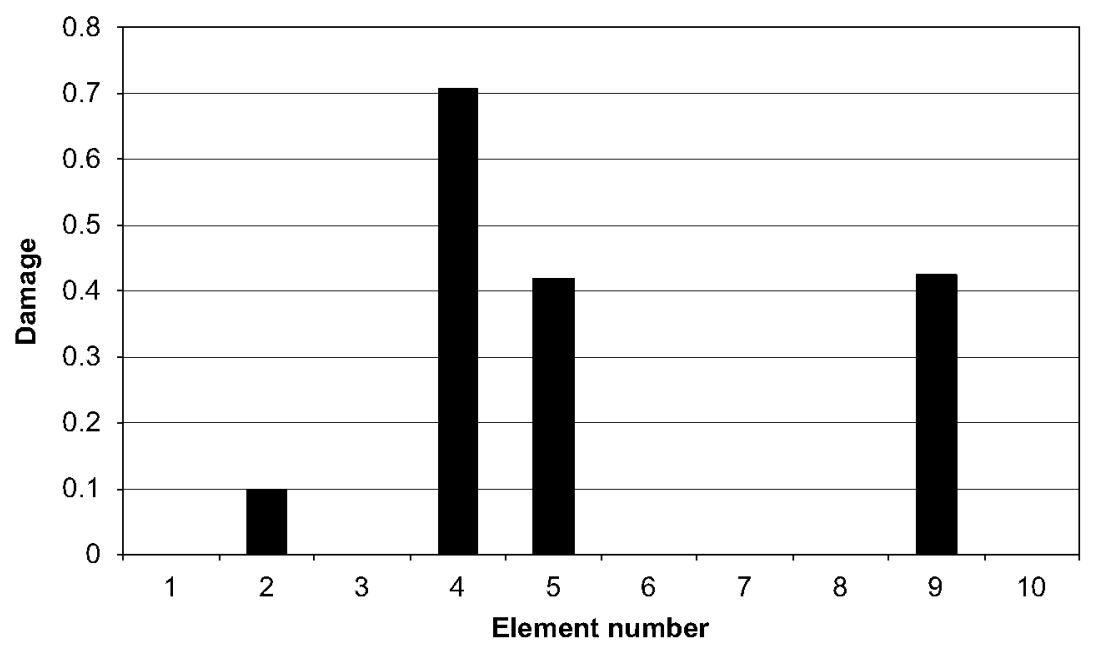

Fig. 22. Midspan damage distribution-I-40 bridge second stage.

\section{Conclusions}

For the first time, a multistage damage identification method applicable to large structural systems and formulated in a multiobjective evolutionary context has been proposed. The proposed hierarchical diagnosis strategy aims at the successive detection of the occurrence and approximate location of structural damage in a first stage and at refined location and extent of damage in a second stage and has been applied with success to numerically simulated structures and to an experimentally tested bridge. The proposed approach makes it possible to apply different numerical and experimental schemes at each stage according to necessity and notably decreases the number of parameters to update at each stage by removing ill-conditioning numerical problems.

Furthermore, to make the damage identification procedure easier two independent objective functions have been formulated to carry out the FE model updating. One of the objective functions is based on modal flexibility because of its high sensitivity to local damage. To choose the other function the main disadvantage of modal flexibility due to its sparseness in damage identification was taken into account; as a result, a function dependent on mode shapes and frequencies, combined in such a way as to be dependent on damage location, was formulated. To avoid the difficulty of weighting the two objective functions in a single-objective function, an evolutionary multiobjective optimization technique was used to find all of the good trade-off solutions available for the problem (the so-called Pareto-optimal set) in a single run of the algorithm. With this approach the computation cost is decreased and non-a priori selection is taken in the optimization procedure. The simulated and real case studies have shown that the proposed methodology is robust, fast and easy to implement.

\section{Acknowledgments}

The writers acknowledge support for the work reported in this paper from the Ministry of Education and Science of Spain (project BIA2004-06272). They are also gratefully acknowledged to Farrar et al. [36] by the experimental data used in this work.

\section{References}

Y.J. Yan, L. Cheng, Z.Y. Wu, L.H. Yam, Development in vibration-based structural damage detection technique, Mechanical Systems and Signal Processing 21 (2007) 2198-2211. 
S.W. Doebling, C.R. Farrar, M.B. Prime, A summary review of vibration-based damage identification methods, Shock and Vibration Digest 30 (2) (1998) 91-105.

N. Bicanic, H. Chen, Damage identification in framed structures using natural frequencies, International Journal of Numerical Methods in Engineering 40 (1997) 4451-4468.

O.S. Salawu, Detection of structural damage through changes in frequency: a review, Engineering Structures 19 (9) (1997) 718-723.

W.X. Ren, G. De Roeck, Structural damage identification using modal data. I: Simulation verification, Journal of Structural Engineering ASCE 128 (1) (2002) 87-95.

W.X. Ren, G. De Roeck, Structural damage identification using modal data. I: Test verification, Journal of Structural Engineering ASCE 128 (1) (2002) 96-104.

S. Salawu, C. Williams, Bridge assessment using forced vibration testing, Journal of Structural Engineering ASCE 121 (2) (1995) 161-172.

J.T. Kim, S.H. Jung, Y.K. Lee, J.W. Yun, Damage identification in bridges using vibration-based system identification scheme, in: Proceedings of 18th International Modal Analysis Conference, San Antonio, TX, 2000, pp. 1327-1333.

A.K. Pandey, M. Biswas, M.M. Samman, Damage detection from changes in curvature mode shapes, Journal of Sound and Vibration 145 (2) (1991) 321-332.

M.M. Abdel Wahab, G. De Roeck, Damage detection in bridges using modal curvatures: application to a real damage scenario, Journal of Sound and Vibration 226 (2) (1999) 217-235.

Z.Y. Shi, S.S. Law, L.M. Zhang, Structural damage detection from modal strain energy change, Journal of Engineering Mechanics ASCE 126 (12) (2000) 1216-1223.

W.X. Ren, G. De Roeck, Discussion on structural damage detection from modal strain energy change, Journal of Engineering Mechanics ASCE 128 (3) (2002) 376-377.

A.K. Pandey, M. Biswas, Damage detection in structures using changes in flexibility, Journal of Sound and Vibration 169 (1994) 3-17.

B. Jaishi, W.X. Ren, Damage detection by finite element model updating using modal flexibility residual, Journal of Sound and Vibration 290 (2006) 369-387.

J.M.W. Brownjohn, P.Q. Xia, H. Hao, Y. Xia, Civil structure condition assessment by FE model updating methodology and case studies, Finite Elements in Analysis and Design 37 (2001) 761-775.

M.I. Friswell, Damage identification using inverse methods, Philosophical Transactions of the Royal Society 365 (1851) (2007) 393-410.

C.B. Yun, E.Y. Bahng, Substructural identification using neural networks, Computers and Structures 77 (2000) 41-52.

J.M. Ko, Z.G. Sun, Y.Q. Ni, Multi-stage identification scheme for detecting damage in cable-stayed Kap Shui Mun Bridge, Engineering Structures 24 (2002) 857-868.

A. Teughels, J. Maeck, G. De Roeck, Damage assessment by FE model updating using damage functions, Computers and Structures 80 (2002) 1869-1879.

C.A. Coello, D.A. Van Veldhuizen, G.B. Lamont, Evolutionary Algorithms for Solving Multi-objective Problems, Kluwer Academic Publishers, New York, USA, 2002.

C.A. Coello, Recent trends in evolutionary multiobjective optimization, evolutionary multiobjective optimization: theoretical advances and applications, in: A. Abraham, L. Jain, R. Goldberg (Eds.), Evolutionary Multiobjective Optimization: Theoretical Advances and Applications, Springer, London, 2005.

J. Lemaitre, A Course on Damage Mechanics, Springer, Berlin, 1996.

M.I. Friswell, J.E.T. Penny, S.D. Garvey, A combined genetic and eigensensitivity algorithm for the location of damage in structures, Computers and Structures 69 (1998) 547-556.

Y. Haralampidis, C. Papadimitriou, M. Pavlidou, Multi-objective framework for structural model identification, Earthquake Engineering and Structural Dynamics 34 (2005) 665-685.

B. Jaishi, W.X. Ren, Structural finite element model updating using ambient vibration test results, Journal of Structural Engineering ASCE 131 (4) (2005) 617-628.

K. Christodoulou, C. Papadimitriou, Structural identification based on optimally weighted modal residuals, Mechanical Systems and Signal Processing 21 (1) (2007) 4-23.

B. Jaishi, W.X. Ren, Finite element model updating based on eigenvalue and strain energy residuals using multiobjective optimisation technique, Mechanical Systems and Signal Processing 21 (5) (2007) 2295-2317.

J. Zhao, J.T. DeWolf, Sensitivity study for vibrational parameters used in damage detection, Journal of Structural Engineering ASCE 125 (4) (1999) 410-416.

R.J. Allemang, D.L. Brown, A correlation for modal vector analysis, in: Proceedings of First International Modal Analysis Conference, 1982, pp. 110-116.

R. Perera, R. Torres, Structural damage detection via modal data with genetic algorithms, Journal of Structural Engineering ASCE 132 (9) (2006) 1491-1501.

J.F. Unger, A. Teughels, G. De Roeck, System identification and damage detection of a prestressed concrete beam, Journal of Structural Engineering ASCE 132 (11) (2006) 1691-1698.

D. Goldberg, Genetic Algorithms in Search, Optimization and Machine Learning, Addison-Wesley, Reading, MA, 1989.

R.I. Levin, N.A.J. Lieven, Dynamic finite element model updating using simulated annealing and genetic algorithms, Mechanical Systems and Signal Processing 12 (1) (1998) 91-120. 
E. Zitzler, L. Thiele, Multiobjective evolutionary algorithms: a comparative case study and the strength Pareto approach, IEEE Transactions on Evolutionary Computation 3 (4) (1999) 257-271.

H. Ahmadian, J.E. Motthershead, M.I. Friswell, Regularisation methods for finite element model updating, Mechanical Systems and Signal Processing 12 (1) (1998) 47-64.

C.R. Farrar, W.E. Baker, T.M. Bell, K.M. Cone, T.W. Darling, T.A. Duffey, A. Eklund, A., Migliori, Dynamic characterization and damage detection in the I-40 bridge over the Rio Grande, Los Alamos National Laboratory Report, LA-12767-MS, 1996.

C.R. Farrar, T.A. Duffey, P.A. Goldman, D.V. Jauregui, J.S. Vigil, Finite element analysis of the I-40 bridge over the Rio Grande, Los Alamos National Laboratory Report, LA-12979-MS, 1996. 